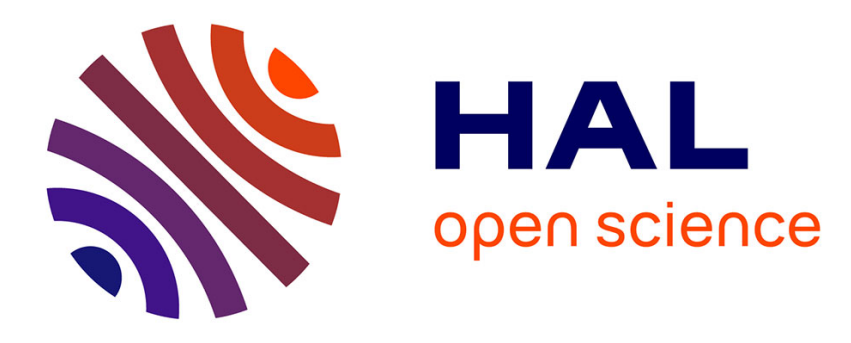

\title{
Etude numérique de la focalisation aléatoire d'une onde se propageant dans une turbulence thermique $2 \mathrm{D}$
}

\author{
Ph. Blanc-Benon, D. Juvé
}

\section{To cite this version:}

$\mathrm{Ph}$. Blanc-Benon, D. Juvé. Etude numérique de la focalisation aléatoire d'une onde se propageant dans une turbulence thermique 2D. Journal de Physique IV Proceedings, 1994, 04 (C5), pp.C5-1001C5-1004. 10.1051/jp4:19945220 . jpa-00252906

\section{HAL Id: jpa-00252906 https://hal.science/jpa-00252906}

Submitted on 1 Jan 1994

HAL is a multi-disciplinary open access archive for the deposit and dissemination of scientific research documents, whether they are published or not. The documents may come from teaching and research institutions in France or abroad, or from public or private research centers.
L'archive ouverte pluridisciplinaire HAL, est destinée au dépôt et à la diffusion de documents scientifiques de niveau recherche, publiés ou non, émanant des établissements d'enseignement et de recherche français ou étrangers, des laboratoires publics ou privés. 


\title{
Etude numérique de la focalisation aléatoire d'une onde se propageant dans une turbulence thermique 2D
}

\author{
Ph. BLANC-BENON et D. JUVÉ
}

Ecole Centrale de Lyon, Laboratoire de Mécanique des Fluides et d'Acoustique, URA 263 du CNRS, ECL, BP. 163, 69131 Ecully cedex, France

\begin{abstract}
We consider the propagation of acoustic waves, in either the geometric or the parabolic approximation, through an ensemble of realizations of a random field modeling a $2 \mathrm{D}$ homogeneous isotropic thermal turbulence. Our results for a plane wave demonstrate that the occurrence of caustics as well as the maximun peak in the scintillation index are governed by the same universal parameter related to the correlation function of the temperature fluctuations.
\end{abstract}

\section{INTRODUCTION}

La propagation des ondes dans les milieux aléatoires est un problème dont l'analyse théorique a été envisagée par de nombreux auteurs ( Tatarski[1], Uscinski[2]) et dont les applications sont nombreuses en acoustique aérienne ou sous-marine (bruit de transports, détection de phénomènes météorologiques, imagerie acoustique, sonar..).Des mesures "in-situ" ainsi que des expériences réalisées en laboratoire ont mis en évidence le rôle important joué entre autre par la turbulence thermique sur la décorrélation de l'onde transmise, sur la déformation des fronts de phase et sur les fluctuations de niveaux sonores ( Blanc-Benon et al.[3]).

Les différentes approches théoriques disponibles dans la littérature ont toutes comme point de départ l'équation stochastique de Helmholtz dans laquelle l'interaction entre l'onde et le milieu est traduite sous la forme d'un indice aléatoire. Les équations moyennées, qui gouvernent l'évolution des moments successifs du champ de pression transmis, sont ensuite résolues en adoptant une hypothèse de fermeture qui repose sur une modèlisation de la fonction de corrélation des fluctuations d'indice ( en général bruit blanc gaussien delta-corrélé suivant la direction de propagation). Si la comparaison avec les résultats expérimentaux est très bonne pour les deux premiers moments, la prédiction des fluctuations d'intensité reste cependant difficile et perfectible.

Nous présentons ici une approche statistique du problème. La turbulence est considérée comme une suite de réalisations indépendantes d'un champ aléatoire obtenu en sommant des modes de Fourier convenablement choisis. Pour chaque réalisation le calcul de propagation acoustique est effectué en utilisant une méthode classique telle que l'acoustique géométrique ou l'approximation parabolique. Les grandeurs statistiques sont ensuites obtenues par moyenne d'ensemble sur un grand nombre de réalisations. De telles simulations sont actuellement réalisées en dimension 2 ou 3 dans le cas d'une turbulence homogène et isotrope, et ceci, aussi bien pour des fluctuations thermiques que des fluctuations cinématiques. Pour le cas d'une turbulence thermique bidimensionnelle, nous analysons ici la focalisation aléatoire d'une onde plane, et nous mettons clairement en évidence 
la relation existant entre la formation d'une zone de caustique et la présence d'un maximun de scintillation pour les fluctuations d'intensité acoustique.

\section{SIMULATION D'UNE TURBULENCE THERMIQUE BIDIMENSIONNELLE HO- MOGENE ET ISOTROPE}

Dans notre étude nous considérons que les structures turbulentes n'évoluent pas durant le temps de traversée de l'onde acoustique et nous faisons l'hypothèse usuelle de turbulence gelée. Le milieu turbulent est alors représenté comme un ensemble de réalisations d'un champ aléatoire. Pour chacune d'entre elles la fluctuation de température $T^{\prime}(\vec{x})$ en un point $\vec{x}$ du plan est obtenue en superposant un nombre fini $\mathrm{N}$ de modes de Fourier aléatoires (Blanc-Benon et al. [4]) soit:

$$
T^{\prime}(\vec{x})=\sum_{j=1}^{N} \mathcal{T}\left(\vec{K}^{j}\right) \cos \left(\vec{K}^{j} \cdot \vec{x}+\phi^{j}\right)
$$

La direction du vecteur d'onde $\vec{K}^{j}$ dans le plan et la phase $\phi^{j}$ sont deux variables aléatoires uniformément distribuées sur l'intervalle $[0,2 \pi]$, de façon à simuler un champ homogène et isotrope. L'amplitude $\mathcal{T}\left(\vec{K}^{j}\right)$ de chaque mode est fixé à partir du spectre $G(K)$ des fluctuations et de la valeur efficace de ces fluctuations $\theta^{2}$. Pour une turbulence bidimensionnelle $G(K)$ est relié à la fonction de corrélation spatiale en deux points $m(r)$ :

$$
G(K)=\theta^{2} K \int_{0}^{\infty} r m(r) J_{0}(K r) d r
$$

( $J_{0}$ est la fonction de Bessel de lère espèce d'ordre zéro). Dans les simulations présentées, la fonction de corrélation est une gaussienne $m(r)=\exp \left(-r^{2} / L^{2}\right)$ et l'échelle intégrale des fluctuations de températures $L_{f}$ a donc pour valeur $L \sqrt{\pi} / 2$. Ici nous avons pris une échelle $L$ de $10 \mathrm{~cm}$ et nous discrétisons le spectre $G(K)$ avec 100 modes de Fourier répartis entre $K_{\min }=0.1 / L$ et $K_{\max }=6 / L$. Les moyennes statistiques sont calculées avec 200 réalisations.

\section{ACOUSTIQUE GEOMETRIQUE}

L'approximation de l'acoustique géométrique, qui correspond à une solution haute fréquence des équations linéarisées de la mécanique des fluides (Candel [ 5 ]), fournit un système d'équations différentielles gouvernant l'évolution des rayons acoustiques ainsi que celle des fronts d'onde. Pour un milieu inhomogène indépendant du temps et d'indice $N=c_{0} / c$, nous obtenons:

$$
\begin{aligned}
\frac{d x_{i}}{d t} & =\frac{c_{0}}{N^{2}} p_{i} \\
\frac{d p_{i}}{d t} & =\frac{c_{0}}{N} \frac{\partial N}{\partial x_{i}} \\
\frac{d R_{i}}{d t} & =\frac{c_{0}}{p N}\left(Q_{i}-\nu_{i} \nu_{j} Q_{j}\right)-\frac{c_{0}}{N^{2}} \nu_{i} R_{j} \frac{\partial N}{\partial x_{j}} \\
\frac{d Q_{i}}{d t} & =\frac{c_{0}}{N} R_{j} \frac{\partial^{2} N}{\partial x_{j} \partial x_{i}}-\frac{c_{0}}{N^{2}} R_{j} \frac{\partial N}{\partial x_{j}} \frac{\partial N}{\partial x_{i}}
\end{aligned}
$$

$\vec{p}$ désigne le vecteur d'onde adimensionnel $\vec{p}=N \vec{\nu}$ tangent à la trajectoire. Dans un cas bidimensionnel, le vecteur position $\vec{x}$ et le vecteur d'onde $\vec{p}$ associés au point courant du rayon sont fonction uniquement du temps de parcours t et de la position sur le front d'onde initial. Pour une onde incidente plane nous avons:

$$
\vec{x}(t=0)=\left(\begin{array}{c}
0 \\
y^{0}
\end{array}\right) \quad \vec{p}(t=0)=N \vec{\nu}(t=0)
$$


Les éléments géodésiques $\vec{R}=\left(\partial \vec{x} / \partial y^{0}\right)_{t}$ et $\vec{Q}=\left(\partial \vec{p} / \partial y^{0}\right)_{t}$ qui gouvernent l'évolution des fronts d'onde orthogonaux aux trajectoires permettent de caractériser la section droite d'un tube élémentaire de rayons. En cherchant les conditions d'annulation de cette section droite, on localise dans le plan les caustiques ce qui permet d'étudier pour un milieu turbulent la densité de probabilité de la distance de formation de la première caustique. Les conditions initiales pour ces deux vecteurs sont:

$$
\vec{R}(t=0)=\left(\begin{array}{l}
0 \\
1
\end{array}\right) \quad \vec{Q}(t=0)=\frac{\partial p(0)}{\partial y^{0}}\left(\begin{array}{l}
1 \\
0
\end{array}\right)
$$

Le système différentiel (3) est intégré avec une méthode de Runge-Kutta d'ordre 4 avec un pas de temps $d t=1 . / c_{0} K_{m a x}$ défini à partir du nombre d'onde turbulent maximum utilisé dans la. modélisation du spectre des fluctuations de température $G(K)$.

\section{APPROXIMATION PARABOLIQUE}

Soit $\mathrm{x}$ la direction privilégiée de propagation, en écrivant la pression acoustique sous la forme $p(\vec{x})=$ $\psi(\vec{x}) \exp \left(i k_{0} x\right)$, on montre que l'enveloppe $\psi(\vec{x})$ est solution de l'équation parabolique:

$$
2 i k_{0} \frac{\partial \psi}{\partial x}+\frac{\partial^{2} \psi}{\partial y^{2}}+k_{0}^{2} \epsilon \psi=0
$$

où les fluctuations aléatoires d'indice $\epsilon$ sont reliées aux fluctuations de températures $\left(\epsilon(\vec{x})=-T^{\prime}(\vec{x}) / T_{0}\right)$. Dans cette approximation les effets de diffraction sont pris en compte mais l'énergie rétrodiffusée est négligée. Pour résoudre cette équation nous utilisons un algorithme "grand angle" fondé sur la transformée de Fourier rapide et développé par Thomson et Chapman [6] pour des applications en acoustique sous-marine. Le champ $\psi$ au pas $\mathrm{x}+\mathrm{h}$ s'exprime en fonction du champ au pas précédent par:

$$
\psi(x+h, y)=\exp \left(i k_{0}(N-1) \mathcal{F}^{-1}\left[\exp \left(-i s^{2} \frac{h}{\left(k_{0}+\sqrt{k_{0}^{2}-s^{2}}\right)}\right) \mathcal{F}(\psi(x, y))\right]\right.
$$

Ici l'indice $N$ est égal à $\epsilon / 2, \mathcal{F}$ désigne la transformée de Fourier suivant y et s est la variable conjuguée de y. Le choix du pas d'intégration h dépend à la fois de la fréquence de l'onde acoustique incidente et de l'échelle de corrélation spatiale des inhomogénéités turbulentes du milieu. Dans les calculs présentés nous avons $h=0,2 L$ et la transformée de Fourier est faite sur 1024 ou 2048 points. De plus pour supprimer les réflexions artificielles introduites par les bords du domaine de calcul nous introduisons une atténuation suivant y portant sur les derniers $10 \%$ des points. Une étude détaillée des conditions d'applications de cet algorithme été faite par Hugon-Jeanin [7].

\section{RESULTATS}

Les deux approches théoriques, dont nous venons de rappeler brièvement le principe, permettent d'analyser la focalisation aléatoire d'une onde plane se propageant dans une turbulence thermique, et de déterminer les relations pouvant exister entre la zone de formation des caustiques et le lieu du maximun des fluctuations d'intensité acoustique.

Sur la figure 1 nous avons tracé la densité de probabilité de formation de la première caustique en fonction de la distance normalisée $s \sigma^{2 / 3}$, où s est la distance de propagation et $\sigma^{2 / 3}$ un paramètre introduit par Kulkarny et White [8]. Lorsque les fluctuations de températures ont une fonction de corrélation gaussienne on montre que $\sigma^{2 / 3}$ a pour valeur $12^{1 / 3}<\mu^{2}>^{1 / 3} \pi^{1 / 6} / L$ avec $\sqrt{\left\langle\mu^{2}\right\rangle}=\theta / 2 T_{0}$ (Blanc-Benon et al. [4]). Pour les résultats présentés ici nous avons $L=10 \mathrm{~cm}$ et $\mu=0,05$. Cette densité de probabilité possède un maximun très marqué pour $s \sigma^{2 / 3}=1,3$, ce qui correspond à la localisation la plus probable de la première caustique. Nous observons le bon accord avec les prédictions de Kulkarny et White. 
Sur la figure 2 nous donnons l'évolution de la variance normalisée des fluctuations d'intensité $\sigma_{I}^{2}=\left(\left\langle I^{2}\right\rangle-\langle I\rangle^{2}\right) /\langle I\rangle^{2}$ en fonction de la distance normalisés $s \sigma^{2 / 3}$. Nous avons tracé trois courbes correspondant à trois valeurs de $\gamma=k_{0}{ }^{2}\left\langle\mu^{2}\right\rangle L^{3} \sqrt{\pi}$ obtenues avec différentes fréquences dans la gamme $40 k H z-80 k H z$. Ce coefficient $\gamma$ ("strength parameter"), qui traduit le rapport entre les effets de diffusion multiple et les effets de distance, permet de caractériser le type des fluctuations d'intensité et notamment le régime de saturation (Uscinski [2]). On observe de façon usuelle que l'indice de scintillation $\sigma_{I}^{2}$ croit avec la distance jusqu'à un maximun avant de diminuer et d'atteindre un palier de saturation. Et le maximun de $\sigma_{I}{ }^{2}$ apparait en moyenne à la même distance normalisée de la source que la première caustique.

\section{CONCLUSION}

Dans l'approximation de l'acoustique géométrique, l'étude de la focalisation d'une onde plane a mis en évidence l'existence d'un paramètre $\sigma^{2 / 3}=12^{1 / 3}<\mu^{2}>^{1 / 3} \pi^{1 / 6} / L$ qui permet de prédire la localisation spatiale la plus probable de zones de caustiques en fonction des caractéristiques de la turbulence. Avec l'approximation parabolique, l'analyse complémentaire de l'évolution de l'indice de scintillation montre clairement le rôle joué par la présence de caustiques dans l'apparition d'un maximun des fluctuations d'intensité acoustique avant le régime de saturation. Cette comparaison confirme l'importance du paramètre $\sigma^{2 / 3}$. Une extension au cas $3 \mathrm{~d}$ est actuellement en cours.

\section{REFERENCES}

[1] Tatarski, V.I., 1971, The effects of the turbulent atmosphere on wave propagation,IPST Keter Press.

[2] Uscinski, B.J., 1985, J. Opt. Soc. Am., A, Vol.2,12, 2077-2091.

[3] Blanc-Benon, Ph., Juvé, D., 1993, Waves in Random Media, Vol.3,71-83.

[4] Blanc-Benon, Ph., Juvé, D., Comte-Bellot, G., 1991, Theor. Comp. Fluid Dyn., Vol.2, 271-278.

[5] Candel, S.M., 1977, J. Fluid Mech. 83, 465-493.

[6] Thomson, D.J., Chapman, N.R., 1983, J. Acous. Soc. Am., Vol. 47, 1848-1854.

[7] Hugon-Jeanin, Y. , 1992, Thèse de Doctorat Ecole Centrale de Lyon 92-37

[8] Kulkarny, V.A., White, B.S., 1982, Phys. Fluids 25, 10, 1770-1784.

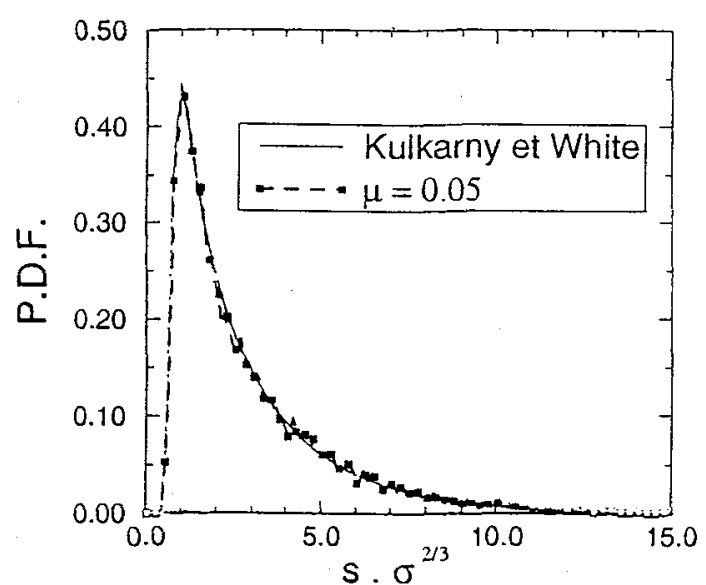

Fig.1. Evolution de la densité de probabilité de la distance de formation de la première caustique avec la distance $s \sigma^{2 / 3}$.

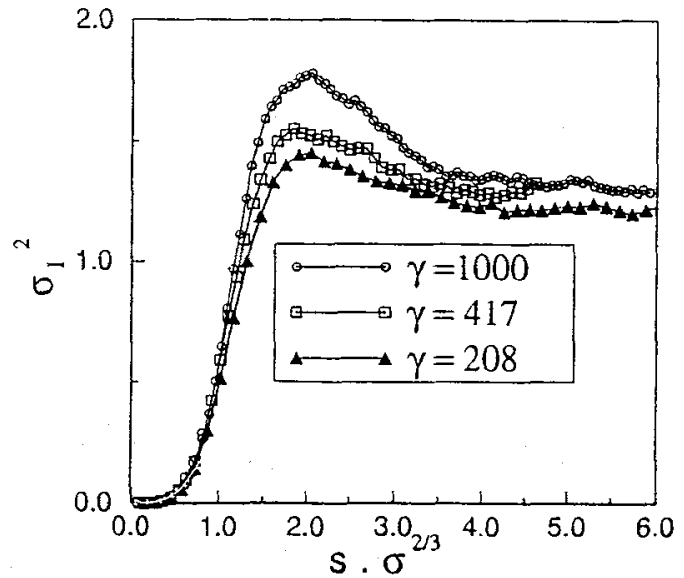

Fig.2. Evolution de la variance des fluctuations d'intensité avec la distance $s \sigma^{2 / 3}$. 